\title{
Acoustic scattering of a complex-source beam by the edge of a plane angular sector
}

\author{
H. Brüns and L. Klinkenbusch \\ Institute of Electrical and Information Engineering, Kiel University, Kaiserstr. 2, 24143 Kiel, Germany \\ Correspondence to: H. Brüns (heb@tf.uni-kiel.de)
}

Received: 7 January 2014 - Revised: 6 May 2014 - Accepted: 27 May 2014 - Published: 10 November 2014

\begin{abstract}
The scattering and diffraction of a complex-source beam (CSB) by an acoustically soft or hard plane angular sector is treated by a rigorous spherical-multipole analysis in sphero-conal coordinates. By assigning a complex-valued radial source coordinate to the corresponding Green's function, the CSB is directed exactly towards the corner of the sector. Since the CSB can be interpreted as a localized plane wave, its interaction with the corner in the presence of the semi-infinite structure can be analyzed in detail. In opposite to the classical case of a non-localized incident plane wave, the resulting multipole series is strongly convergent and no summation techniques are necessary to obtain meaningful results. The numerical results include a convergence analysis, total near fields as well as scattered far fields and prove the applicability of this new approach.
\end{abstract}

\section{Introduction}

Among the canonical problems in electromagnetic scattering theory (see Bowman et al., 1987 for a comprehensive overview) those ones with semi-infinite structures turn out to be rather complicated to solve. Even though analytic results for the diffracted (total) fields are given for a large variety of such geometries (for example in the form of the related Green's functions), it can be a considerably pretentious task to derive the scattered field, in particular if both the source point and the observation point are far away from the definite end (e.g., the tip) of the semi-infinite structure. An example for the successful treatment of such a problem is the wedge and its special case, the half plane. The common way to tackle this problem is to transform the modal (multipole) expansion of the related Green's function into a suitable contour integral in the complex plane and to evaluate the integral asymptotically by means of a steepest-descend or stationaryphase method. This way, one can separate from the total field the incident, reflected and transmitted Geometrical-Optics (GO) terms and obtain a closed-form result for the field caused by the edge. Since the latter part serves as an extension of the conventional GO field, this method is referred to as the Geometrical Theory of Diffraction (GTD, see Keller, 1959, 1962), while its generalization is the Uniform Theory of Diffraction (UTD, see Kouyoumjian and Pathak, 1974). In each case, the edge-related field part is described by the GTD (UTD) diffraction coefficient.

The attempt to apply similar methods to obtain diffraction coefficients for the plane angular sector and especially for the quarter plane was not successful, mainly because of the more complicated structure of the eigenfunction expansion of the related Green's function. The interest in such geometries is -among others- motivated by the fact that they possess a tip, and the related tip-diffraction coefficient could be a further element to complete the GTD. Early work on the diffraction of scalar (acoustic) waves by a plane angular sector goes back to Kraus (1955) and Radlow (1961). While Radlow attempted to tackle the problem by means of a Wiener-Hopf type integral equation approach, Kraus started from a modal expansion of the field in sphero-conal (conical) coordinates in which the sector is described by a coordinate surface. Moreover sphero-conal coordinates belong to those coordinate systems where the Helmholtz equation is fully separable (Eisenhart, 1934), (Moon and Spencer, 1971). The corresponding electromagnetic diffraction problem has been (probably) first treated by Satterwhite (1969). He derived the eigenfunction expansion of the dyadic Green's function of the sector in terms of vector spherical-multipole functions in sphero-conal coordinates. Jansen (1976) achieved a considerable progress in the theory of Lamé functions which are 


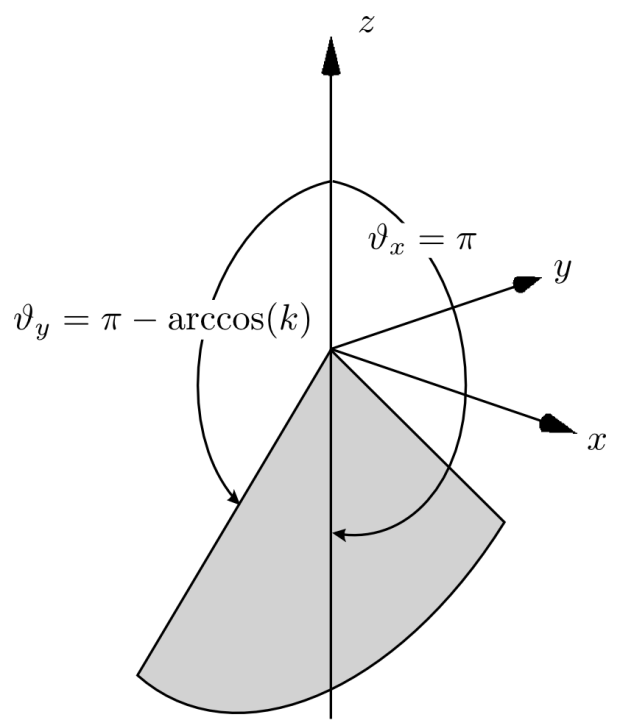

Figure 1. Plane angular sector described by $\vartheta=\pi$ and $k^{2}=0.5$.

part of the elementary solutions of the Helmholtz equation in sphero-conal coordinates. Using these new representations Blume et al. investigated the diffraction by an elliptic cone (including the sector) and found expressions for the related nose-on radar cross sections (RCS) (Blume et al., 1993).

With the eigenfunction expansion it may be relatively easy to rigorously evaluate the diffracted field if the source or the observation point (or both) are located not too far away from the sector's tip, however the obtained series do not converge if both are in the far field. In that case special sequence transformations had to been used to come to the related limiting values. Blume et al. applied such techniques to calculate radar cross sections (RCS) and diffraction coefficients for the elliptic cone and for the sector (Blume and Klinkenbusch, 1999). However, the convergence properties were rather poor so that linear (Klinkenbusch, 2007) as well as non-linear transformation methods had to be employed.

Among the approximate methods, Hansen (1991) deduced corner diffraction coefficients from numerically computed surface currents on a quarter plane, Hill (1990) calculated UTD diffraction coefficients by an asymptotically valid evaluation of the Kirchhoff integral from edge-corrected Physical-Optics currents on a sector, and Maci et al. derived corresponding results by applying the Incremental Theory of Diffraction - ITD (Maci et al., 1994, 1998).

The present approach extends the multipole-based method which allows to derive an expression for the exact scattered far-field of the plane angular sector. The basic new idea is to illuminate just the corner of the sector by means of a complex-source beam (CSB). Such a CSB can be interpreted as a localized plane wave, and thus its interaction with the corner in the presence of the semi-infinite structure can be analyzed in detail. Particularly, the scattered field obtained for such an incident CSB is caused by the area around the

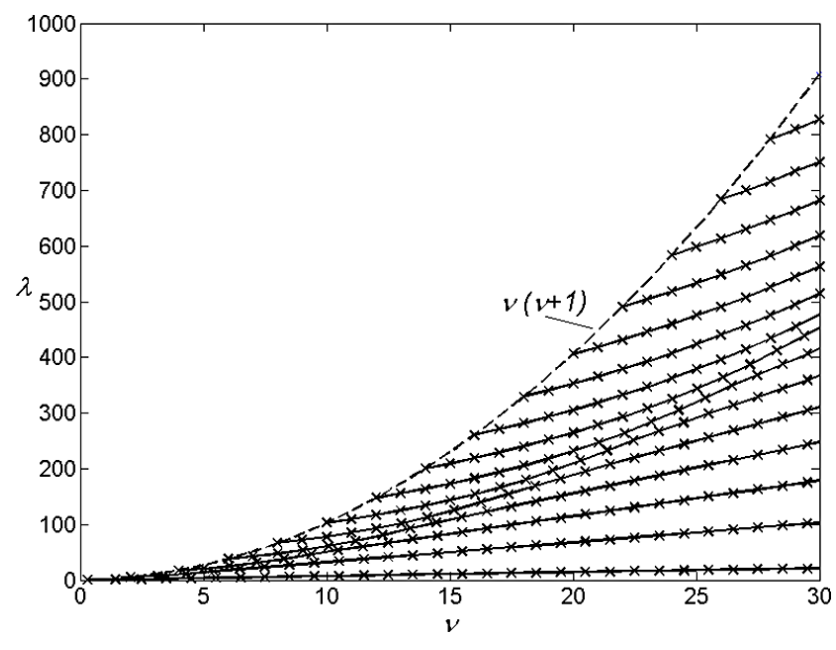

Figure 2. Eigenvalue curves $\lambda(v)$ for all possible solutions of the differential Eq. (9) and discrete Dirichlet eigenvalues $(x)$ satisfying the condition (11) at $\vartheta_{0}=180^{\circ}$ for $k^{2}=0.5$ (quarterplane); function type 1, see Eqs. (14) and (18).

corner only and thus perfectly suited for extending asymptotic methods like the GTD or UTD. Finally, in advantage to the classical case of a full incident plane wave the illumination by a CSB yields a strongly convergent solution, so that the application of series transformation methods as mentioned above is not necessary.

In Sect. 2 and 3 we briefly describe the system of spheroconal coordinates and the solution of the Helmholtz equation in the presence of a plane angular sector, respectively. Section 4 deals with the diffraction of a complex-source beam by the sector, and in Sect. 5 the convergence properties of the method are described. Finally, Sect. 6 numerical results for the diffracted and scattered fields are presented.

\section{Sphero-conal coordinates}

Sphero-conal coordinates $r, \vartheta, \varphi$ are related to Cartesian coordinates by

$$
\begin{aligned}
& x=r \sin \vartheta \cos \varphi \\
& y=r \sqrt{1-k^{2} \cos ^{2} \vartheta} \sin \varphi \\
& z=r \cos \vartheta \sqrt{1-k^{\prime 2} \sin ^{2} \varphi}
\end{aligned}
$$

where $0 \leq r, 0 \leq \vartheta \leq \pi, 0 \leq \varphi \leq 2 \pi$. The so-called geometric parameters $k$ and $k^{\prime}$ are nonnegative real numbers and satisfy $k^{2}+k^{\prime 2}=1$. For $k=1\left(k^{\prime}=0\right)$ sphero-conal coordinates turn into normal spherical coordinates with the $z$ axis as the polar-axis. The normalized metric scaling coefficients 
of the sphero-conal coordinates are given by

$s_{\vartheta}=\frac{1}{r}\left|\frac{\partial \boldsymbol{r}}{\partial \vartheta}\right|=\sqrt{\frac{k^{2} \sin ^{2} \vartheta+k^{\prime 2} \cos ^{2} \varphi}{1-k^{2} \cos ^{2} \vartheta}}$

$s_{\varphi}=\frac{1}{r}\left|\frac{\partial \boldsymbol{r}}{\partial \varphi}\right|=\sqrt{\frac{k^{2} \sin ^{2} \vartheta+k^{\prime 2} \cos ^{2} \varphi}{1-k^{\prime 2} \sin ^{2} \varphi}}$.

The coordinate surface $\vartheta=\vartheta_{0}$ represents a semi-infinite elliptic cone around the $z$ axis (Blume and Klinkenbusch, 1999) which turns into a circular cone for $k=1$. For $\vartheta_{0}=\pi$ the elliptic cone becomes a plane angular sector lying in the $y z$ plane around the negative $z$ axis, see Fig. 1 . The half outer opening angles of that sector are specified by

$\vartheta_{x}=\pi$

$\vartheta_{y}=\pi-\arccos (k)$.

\section{Solution of the Helmholtz equation in the presence of an acoustically soft or hard plane angular sector}

Sphero-conal coordinates count to those eleven coordinates systems described by Eisenhart (1934) where for the threedimensional Helmholtz equation

$\Delta \Psi(r, \vartheta, \varphi)+\kappa^{2} \Psi(r, \vartheta, \varphi)=0$

the method of separation is completely successful. In Eq. (6), the wave number $\kappa$ is related to the wavelength $\Lambda$ by $\kappa=$ $2 \pi / \Lambda$. The resulting elementary solution can be written as

$\Psi_{v}(r, \vartheta, \varphi)=z_{v}(\kappa r) \Theta_{v}(\vartheta) \Phi_{v}(\varphi)$

with spherical Bessel functions being related by

$z_{v}(\kappa r)=\sqrt{\frac{\pi}{2 \kappa r}} Z_{v+1 / 2}(\kappa r)$

to ordinary Bessel functions $Z$ of order $v+1 / 2$. The periodic Lamé functions $\Phi_{\nu}(\varphi)$ and the non-periodic Lamé functions $\Theta_{\nu}(\vartheta)$ satisfy the differential equations

$$
\begin{gathered}
\sqrt{1-k^{\prime 2} \sin ^{2} \varphi} \frac{d}{d \varphi}\left(\sqrt{\left.1-k^{\prime 2} \sin ^{2} \varphi \frac{d \Phi_{v}}{d \varphi}\right)}\right. \\
+\left[\lambda-v(v+1) k^{\prime 2} \sin ^{2} \varphi\right] \Phi_{\nu}=0 \\
\sqrt{1-k^{2} \cos ^{2} \vartheta} \frac{d}{d \vartheta}\left(\sqrt{1-k^{2} \cos ^{2} \vartheta} \frac{d \Theta_{v}}{d \vartheta}\right) \\
+\left[v(v+1)\left(1-k^{2} \cos ^{2} \vartheta\right)-\lambda\right] \Theta_{v}=0
\end{gathered}
$$

Equations (9) and (10) are coupled by the two separation constants $v$ and $\lambda$ which also can be interpreted as eigenvalues of a two-parametric eigenvalue problem. For the problem

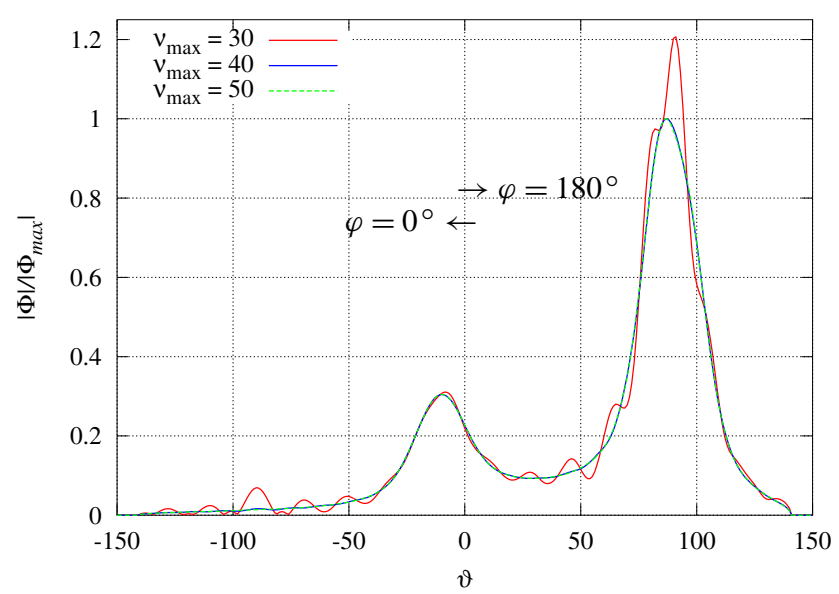

Figure 3. Radiation patterns of an acoustically soft sector $\left(k^{2}=\right.$ $0.6)$ in the $x z$ plane $\left(\varphi=0^{\circ}\right.$ and $\left.\varphi=180^{\circ}\right)$ for different maximum numbers of eigenfunctions. The point source is located at the complex coordinates $r_{c}=(0.001+10 j) \Lambda, \vartheta_{c}=90^{\circ}, \varphi_{c}=90^{\circ}$, that is $\boldsymbol{r}_{c}=(0.001+10 j) \Lambda \hat{y}$ in Cartesian coordinates.

under consideration the periodic Lamé functions have to be $2 \pi$ periodic, and for any such solution the eigenvalues in the $(\nu, \lambda)$-plane lie on so-called eigenvalue curves, the courses of which depend only on $k^{2}$ (Blume and Klinkenbusch, 1999), see Fig. 2. To satisfy the boundary-value problem posed by the acoustically soft or hard plane-angular sector, the nonperiodic Lamé functions have to satisfy either the Dirichlet (acoustically soft, index $s$ ) or the Neumann (acoustically hard, index $h$ ) boundary condition on the sector's surface at $\vartheta=\pi$ :

$\Theta_{v_{s}}(\pi)=0$

$\left.\frac{d \Theta_{v_{h}}}{d \vartheta}\right|_{\vartheta=\pi}=0$.

The corresponding $\left(v_{\tau}, \lambda\right) ; \tau \in\{s, h\}$ eigenvalue pairs are then discrete Dirichlet and Neumann eigenvalues on the eigenvalue curves as shown in Fig. 2. As in ordinary spherical coordinates, for solutions in the free space all eigenvalues $v$ are all non-negative integers $(v=n=0,1,2,3, \ldots)$ on these eigenvalue curves.

The products of non-periodic and periodic Lamé functions are referred to as the Lamé products. They can be represented by a linear combination of four appropriately defined function types

$Y_{v}^{(l)}(\vartheta, \varphi)=\Theta_{v}^{(l)}(\vartheta) \Phi_{v}^{(l)}(\varphi) \quad(l=1 ; 2 ; 3 ; 4)$. 

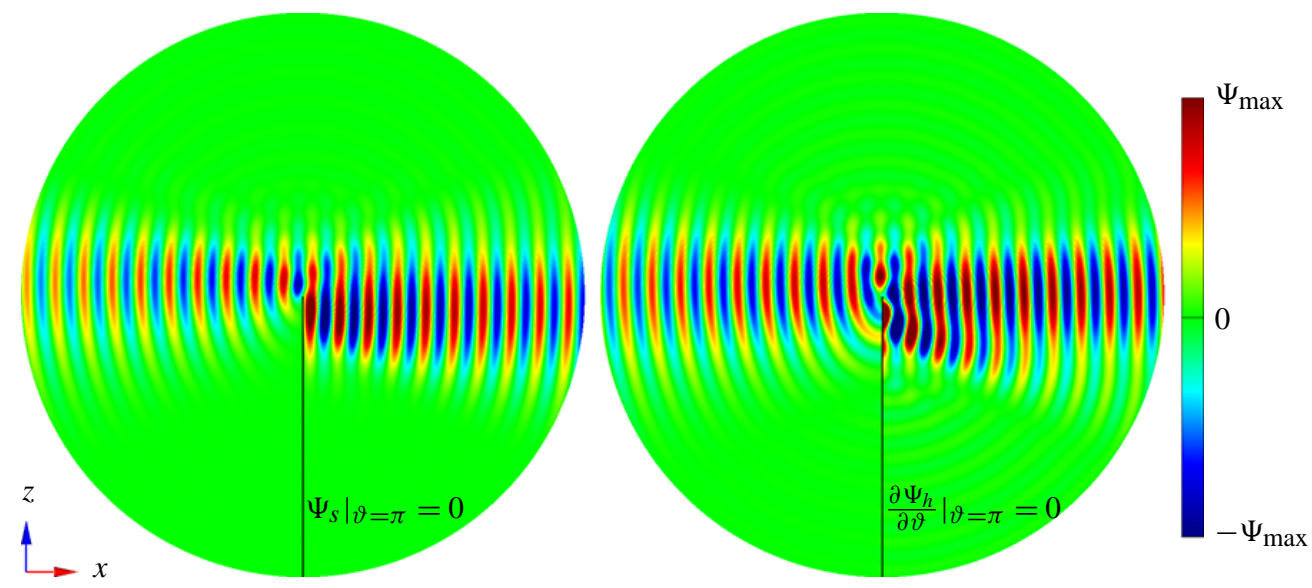

Figure 4. Real part of the total field in the $x z$ plane near the edge $(r<10 \Lambda)$ for an acoustically soft (left-hand side) and acoustically hard (right-hand side) sector. The point source has the complex location $r_{c}=(0.001+10 j) \Lambda, \vartheta_{c}=90^{\circ}, \varphi_{c}=0^{\circ}$, that is $\boldsymbol{r}_{c}=(0.001+10 j) \Lambda \hat{x}$ in Cartesian coordinates.

The four types of periodic Lamé functions are described by each two alternatively valid Fourier series (Jansen, 1976)

$$
\begin{aligned}
& \Phi_{\nu}^{(1)}(\varphi)=\left\{\begin{array}{l}
\sum_{i=0}^{\infty} A_{2 i} \cos 2 i \varphi \\
\sqrt{1-k^{\prime 2} \sin ^{2} \varphi} \sum_{i=0}^{\infty} C_{2 i} \cos 2 i \varphi
\end{array}\right. \\
& \Phi_{\nu}^{(2)}(\varphi)=\left\{\begin{array}{l}
\sum_{i=0}^{\infty} A_{2 i+1} \cos (2 i+1) \varphi \\
\sqrt{1-k^{\prime 2} \sin ^{2} \varphi} \sum_{i=0}^{\infty} C_{2 i+1} \cos (2 i+1) \varphi
\end{array}\right. \\
& \Phi_{\nu}^{(3)}(\varphi)=\left\{\begin{array}{l}
\sum_{i=0}^{\infty} B_{2 i+2} \sin (2 i+2) \varphi \\
\sqrt{1-k^{\prime 2} \sin 2} \varphi \sum_{i=0}^{\infty} D_{2 i+2} \sin (2 i+2) \varphi
\end{array}\right. \\
& \Phi_{\nu}^{(4)}(\varphi)=\left\{\begin{array}{l}
\sum_{i=0}^{\infty} B_{2 i+1} \sin ^{\infty}(2 i+1) \varphi \\
\sqrt{1-k^{\prime 2} \sin ^{2} \varphi} \sum_{i=0}^{\infty} D_{2 i+1} \sin (2 i+1) \varphi .
\end{array}\right.
\end{aligned}
$$

The non-periodic Lamé functions are expanded in terms of associated Legendre functions of the first kind (Jansen, 1976)

$$
\begin{aligned}
& \Theta_{v}^{(1)}(\vartheta)=\sum_{i=0}^{\infty} A_{2 i} T(2 i) P_{\nu}^{2 i}(\cos \vartheta) \\
& \Theta_{v}^{(2)}(\vartheta)=\sum_{i=0}^{\infty} A_{2 i+1} T(2 i+1) P_{v}^{2 i+1}(\cos \vartheta) \\
& \Theta_{v}^{(3)}(\vartheta)=\frac{\sqrt{1-k^{2} \cos ^{2} \vartheta}}{\sin \vartheta} \\
& \sum_{i=0}^{\infty} B_{2 i+2}(2 i+2) T(2 i+2) P_{v}^{2 i+2}(\cos \vartheta)
\end{aligned}
$$

$$
\begin{aligned}
& \Theta_{v}^{(4)}(\vartheta)=\frac{\sqrt{1-k^{2} \cos ^{2} \vartheta}}{\sin \vartheta} \\
& \sum_{i=0}^{\infty} B_{2 i+1}(2 i+1) T(2 i+1) P_{\nu}^{2 i+1}(\cos \vartheta)
\end{aligned}
$$

where the algebraic factors $T(i)$ are recursively defined as

$$
\begin{aligned}
& T(i)=-(v-1)(v+i+1) T(i+2) \\
& T(0)=T(1)=1 .
\end{aligned}
$$

For the method of finding the coefficients we refer to Blume and Klinkenbusch (1999).

For integer eigenvalues $v=n=1,2,3, \ldots$ (free-space solutions) it has been shown (Jansen, 1976) that for each $n$ always one expansion per function type in Eqs. (14)-(17) becomes finite while the expansions (18)-(21) all are finite. Moreover, for each integral eigenvalue $n$ there exist $2 n+1$ eigenfunctions in total, distributed among the function types. In that case Lamé functions turn into Lamé polynomials denoted by $\Phi_{n, m}^{(l)}$ and $\Theta_{n, m}^{(l)}$, where $m$ stands for one of the $2 n+1$ linearly independent eigenfunctions per $n$.

Finally, the summation of all suitable elementary solutions (7) leads to the complete field representation in the domain outside of the acoustically soft or hard plane angular sector. Similar to the method described by Bowman et al. (1987), at a time factor $e^{j \omega t}$ for a unit point source located at $\boldsymbol{r}^{\prime}$ the solution can be written as a symmetric bilinear expansion according to

$\Psi_{\tau}(\boldsymbol{r})=j \kappa \sum_{\nu} j_{v_{\tau}}\left(\kappa r_{<}\right) h_{\nu_{\tau}}^{(2)}\left(\kappa r_{>}\right) Y_{\nu_{\tau}}(\vartheta, \varphi) Y_{\nu_{\tau}}\left(\vartheta^{\prime}, \varphi^{\prime}\right)$

where $r_{<}=r, r_{>}=r^{\prime}$ if $|r|<\left|r^{\prime}\right|$, and $r_{<}=r^{\prime}, r_{>}=r$ if $|r|>\left|r^{\prime}\right| ; \tau \in\{s, h\}$. 

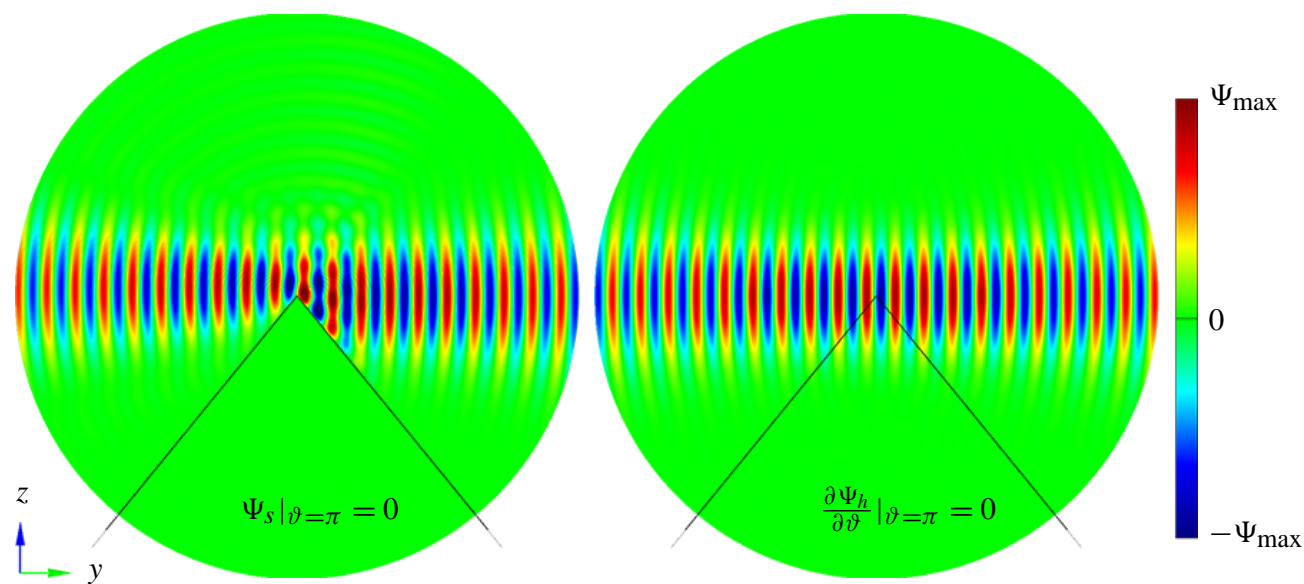

Figure 5. Real part of the total field in the $y z$ plane near the edge $(r<10 \Lambda)$ for an acoustically soft (left-hand side) and acoustically hard (right-hand side) sector. The point source has the complex location $r_{c}=(0.001+10 j) \Lambda, \vartheta_{c}=90^{\circ}, \varphi_{c}=90^{\circ}$, that is $\boldsymbol{r}_{c}=(0.001+10 j) \Lambda \hat{y}$ in Cartesian coordinates.

\section{Complex-source beam incident on an acoustically soft or hard plane angular sector}

A complex-source beam (CSB) is a powerful tool to describe focused beams analytically. As shown by (Katsav et al., 2012) and (Brüns and Klinkenbusch, 2013), the Green's function of a point source at a complex-valued location can be used to approximately describe a Gaussian beam with an arbitrary beam direction. However, in contrast to a Gaussian beam, the CSB exactly satisfies the Helmholtz equation.

Let the complex valued location $\boldsymbol{r}_{c}$ of such a point source be defined by

$\boldsymbol{r}_{c}=\boldsymbol{r}_{r}-j \boldsymbol{b}$.

Then the vector $\boldsymbol{r}_{r}$ represents the location of the beam's waist while $\boldsymbol{b} /|\boldsymbol{b}|$ specifies the direction of propagation. $|\boldsymbol{b}|$ corresponds to the Rayleigh length of the beam, that is, to the distance between the waist and the area where the cross-section has doubled (Brüns and Klinkenbusch, 2013). Consequently, by choosing small $r_{r}$ and large $b$ we obtain a nearly plane wave front at the origin (e.g., at the corner of the plane angular sector). In addition, that field has a transverse Gaussian profile thus the CSB represents a localized plane wave.

As shown by Katsav et al. (2012) a CSB pointing directly to the origin is defined by simply assigning a complex radial coordinate $r_{c}=r_{r}+j b$ to the source. By means of a quite similar procedure we can easily prove that for sphero-conal coordinates as well: Consider that the waist of the CSB is located at $\boldsymbol{r}_{r}$, and that a vector pointing from $\boldsymbol{r}_{r}$ to the origin is given by $\boldsymbol{b}=\left(b, \pi-\vartheta_{r}, \pi+\vartheta_{r}\right)$. According to Eq. (24) and by using Eq. (1) we write in Cartesian coordinates:

$$
\begin{aligned}
\boldsymbol{r}_{c}= & \boldsymbol{r}_{r}-j \boldsymbol{b} \\
= & \left(\begin{array}{c}
r_{r} \sin \vartheta_{r} \cos \varphi_{r} \\
r_{r} \sqrt{1-k^{2} \cos ^{2}\left(\vartheta_{r}\right)} \sin \varphi_{r} \\
r_{r} \cos \vartheta_{r} \sqrt{1-k^{\prime 2} \sin ^{2} \varphi_{r}}
\end{array}\right) \\
& -j\left(\begin{array}{c}
b \sin \left(\pi-\vartheta_{r}\right) \cos \left(\pi+\varphi_{r}\right) \\
b \sqrt{1-k^{2} \cos ^{2}\left(\pi-\vartheta_{r}\right)} \sin \left(\pi+\varphi_{r}\right) \\
b \cos \left(\pi-\vartheta_{r}\right) \sqrt{1-k^{\prime 2} \sin ^{2}\left(\pi+\varphi_{r}\right)}
\end{array}\right) \\
= & \left(\begin{array}{c}
\left(r_{r}+j b\right) \sin \vartheta_{r} \cos \varphi_{r} \\
\left(r_{r}+j b\right) \sqrt{1-k^{2} \cos ^{2} \vartheta_{r}} \sin \varphi_{r} \\
\left(r_{r}+j b\right) \cos \vartheta_{r} \sqrt{1-k^{\prime 2} \sin ^{2} \varphi_{r}}
\end{array}\right)
\end{aligned}
$$

Consequently, by just assigning a complex valued source point in sphero-conal coordinates $r_{c}=r_{r}+j b, \vartheta_{c}=\vartheta_{r}, \varphi_{c}=$ $\varphi_{r}$ we obtain a CSB with a waist at $\boldsymbol{r}_{r}$ and Rayleigh length $b$ directed exactly towards the corner of the plane angular sector.

Now we assign such a complex valued location in spheroconal coordinates $r^{\prime}=r_{c}=r_{r}+j r_{i}, \vartheta_{c}=\vartheta_{r}, \varphi_{c}=\varphi_{r}$ to the point source in Eq. (23), and obtain the total field of an acoustically soft or hard sector illuminated by a complex-source beam directed exactly towards its tip.

Finally it is noted that the incident CSB field (the field of the same CSB point source at $\boldsymbol{r}_{c}$ as in Eq. (23) but in the absence of the sector) is expanded in terms of Lamé polynomials according to

$$
\Psi^{i n c}(\boldsymbol{r})=j \kappa \sum_{n, m} j_{n}\left(\kappa r_{<}\right) h_{n}^{(2)}\left(\kappa r_{>}\right) Y_{n, m}(\vartheta, \varphi) Y_{n, m}\left(\vartheta^{\prime}, \varphi^{\prime}\right)
$$

The scattered field is then obtained by

$$
\Psi_{\tau}^{s c}(\boldsymbol{r})=\Psi_{\tau}(\boldsymbol{r})-\Psi^{i n c}(\boldsymbol{r}) \quad(\tau \in\{s, h\}) .
$$




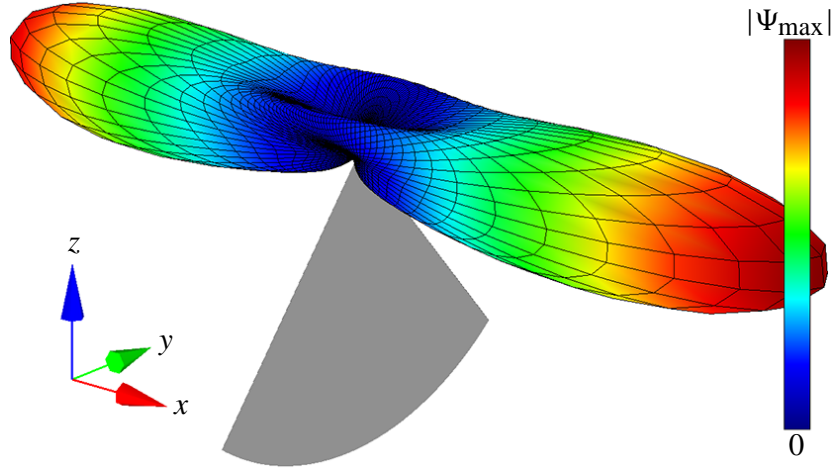

Figure 6. Radiation pattern of the scattered far field for an acoustically soft sector. The source has the complex location $r_{c}=(0.001+$ $10 j) \Lambda, \vartheta_{c}=90^{\circ}, \varphi_{c}=0^{\circ}$, that is $\boldsymbol{r}_{c}=(0.001+10 j) \Lambda \hat{x}$ in Cartesian coordinates.

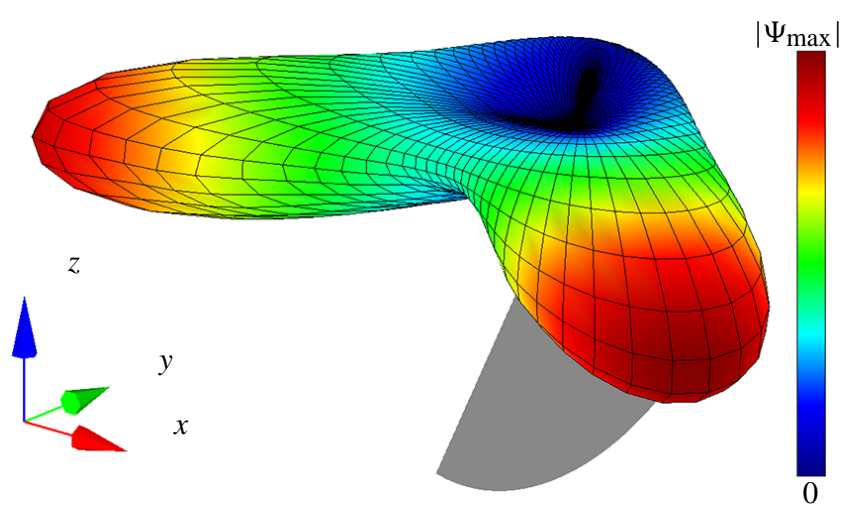

Figure 7. Radiation pattern of the scattered far field for an acoustically soft sector. The source has the complex location $r_{c}=(0.001+$ $10 j) \Lambda, \vartheta_{c}=90^{\circ}, \varphi_{c}=45^{\circ}$, that is $\boldsymbol{r}_{c}=(0.001+10 j) \Lambda\left(\frac{1}{\sqrt{2}} \hat{x}+\right.$ $\left.\frac{1}{\sqrt{2}} \hat{y}\right)$ in Cartesian coordinates.

\section{Convergence analysis}

Basically, the convergence of the expansion (23) is determined by the behaviour of the spherical Bessel functions for a fixed argument and large values of the order $v$ (see Abramowitz and Stegun, 1972, Eq. (9.3.1.) using the definition (8). Note that in Eq. (23) the argument of the spherical Bessel function of the first kind is always smaller than the argument of the spherical Hankel function of the second kind, and hence for any limited and fixed arguments the product of both functions becomes arbitrarily small for any order larger than a certain fixed limit. Consequently, to achieve a given accuracy of the expansion (23) it is sufficient to consider all eigenvalue pairs $(v ; \lambda)$ up to a certain $v \leq v_{\max }$. Table 1 shows the maximum value $v_{\max }$ needed for achieving a relative error of the field expansion (23) for different values of the angular position of the CSB waist $\left(\varphi_{r}\right)$ and of the Rayleigh length $(b)$. As expected the Rayleigh length influences the maximum eigenvalue $v_{\max }$ needed to obtain a cer-

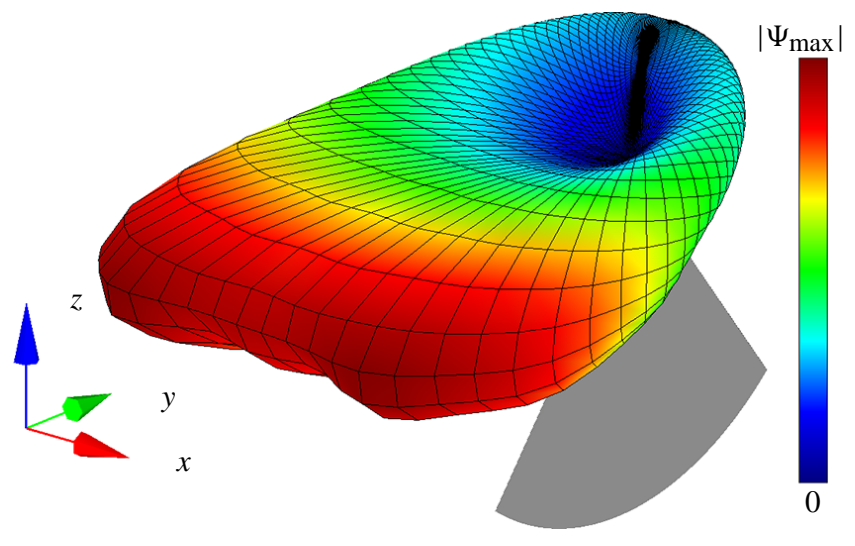

Figure 8. Radiation pattern of the scattered far field for an acoustically soft sector. The source has the complex location $r_{c}=$ $(0.001+10 j) \Lambda, \vartheta_{c}=90^{\circ}, \varphi_{c}=90^{\circ}$, that is $\boldsymbol{r}_{c}=(0.001+10 j) \Lambda \hat{y}$ in Cartesian coordinates.

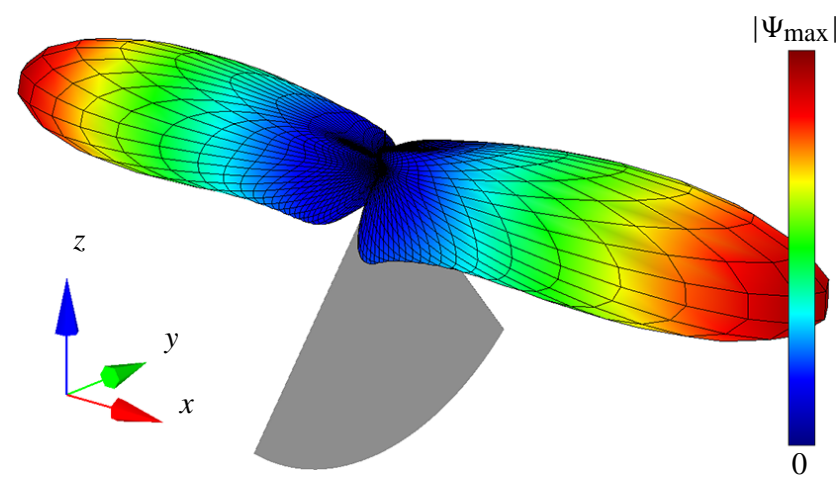

Figure 9. Radiation pattern of the scattered far field for an acoustically hard sector. The source has the complex location $r_{c}=$ $(0.001+10 j) \Lambda, \vartheta_{c}=90^{\circ}, \varphi_{c}=0^{\circ}$, that is $\boldsymbol{r}_{c}=(0.001+10 j) \Lambda \hat{x}$ in Cartesian coordinates.

tain accuracy of Eq. (23) while the angle of incidence $\varphi_{r}$ does not essentially affect the convergence properties.

To check the convergence of the spherical-multipole expansion (23) with regard to the following numerical evaluation, Fig. 3 shows the radiation pattern of the scattered far field in the $x z$ plane with a source located at $r_{c}=(0.001+$ $10 \mathrm{j}) \Lambda, \vartheta_{c}=90^{\circ}, \varphi_{c}=90^{\circ}$. The outer half opening angle of the sector is $\vartheta_{y}=140.77^{\circ}$. The expansion of the field is obtained for different maximum numbers of eigenfunctions, i.e., $v_{\max }=30,40$ and 50. The results are normalized with respect to the maximum of $\Psi_{\tau}^{s c}$ at $v_{\max }=50$. As can be observed, for a maximum number of $v_{\max }=40$ the multipole expansion is convergent for this example. Thus for the parameters chosen in this investigation it is sufficient to carry out the expansion to a maximum number of $\nu_{\max }=40$. 


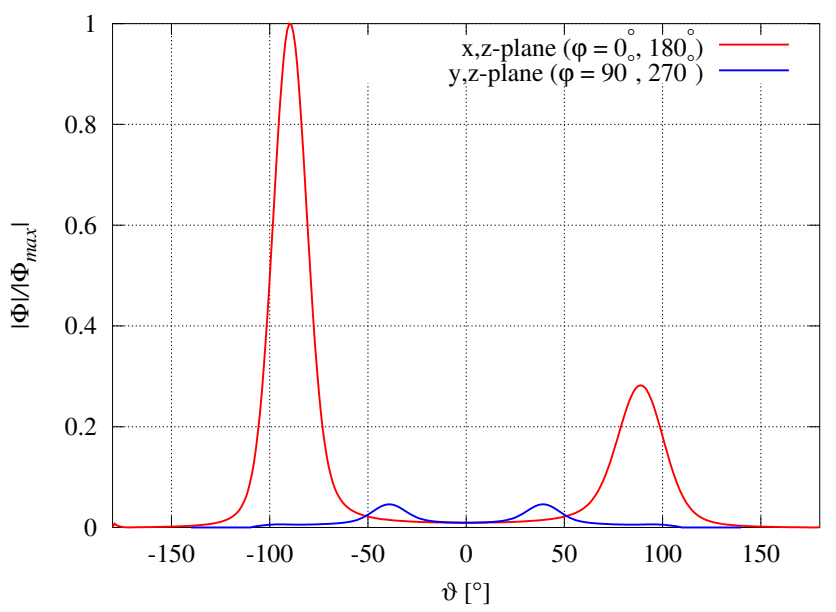

Figure 10. Radiation pattern of the scattered far field for an acoustically soft sector in the $x z$ and $y z$ plane. The source has the complex location $r_{c}=(0.001+10 j) \Lambda, \vartheta_{c}=90^{\circ}, \varphi_{c}=0^{\circ}$, that is $\boldsymbol{r}_{c}=(0.001+10 j) \Lambda \hat{x}$ in Cartesian coordinates.

Table 1. Maximum eigenvalue $v_{\max }$ necessary to achieve a relative error $<10^{-6}$ in (23) for different Rayleigh lengths $r_{i}$ and angles of incidence $\varphi^{\prime}$.

\begin{tabular}{l|l|l|l}
\hline & $b / \Lambda=5$ & $b / \Lambda=10$ & $b / \Lambda=15$ \\
\hline$\varphi_{r}=0^{\circ}$ & 32.00 & 45.00 & 55.00 \\
$\varphi_{r}=45^{\circ}$ & 32.01 & 45.05 & 55.09 \\
$\varphi_{r}=90^{\circ}$ & 32.01 & 45.05 & 55.09 \\
\hline
\end{tabular}

\section{Numerical evaluation}

For the following results we set $k^{2}=0.6$. According to Eq. (5) this yields a plane angular sector with a half outer opening angle of $\vartheta_{y}=140.77^{\circ}$. Moreover, the complex location of the source is lying in the $x y$ plane $\left(\vartheta_{c}=90^{\circ}\right)$ at $r_{c}=(0.001+10 j) \Lambda$. The real part $r_{r}$ is relatively small to take advantage of the beam's property of a nearly plane wave front nearby the waist.

\subsection{Total fields}

The results in Figs. 4 and 5 show the total field $\Psi_{\tau}$ in the nearfield range $r<10 \Lambda$ in the $x z$ plane (Fig. 4) and in the $y z$ plane (Fig. 5). For each of the figures the results on the lefthand side are for an acoustically soft sector while the results on the right-hand side are for an acoustically hard sector.

In Fig. 4 the source has the complex-valued location $r_{c}=$ $(0.001+10 j) \Lambda, \vartheta_{c}=90^{\circ}, \varphi_{c}=0^{\circ}$, that is, the beam is propagating towards the corner in a plane of incidence perpendicular to the sector. In both cases (soft and hard) we observe interferences of reflected and incident fields on the right-hand side of the sector.

In Fig. 5 the source is located at the complex coordinates $r_{c}=(0.001+10 j) \Lambda, \vartheta_{c}=90^{\circ}, \varphi^{\prime}=90^{\circ}$, that is, the beam

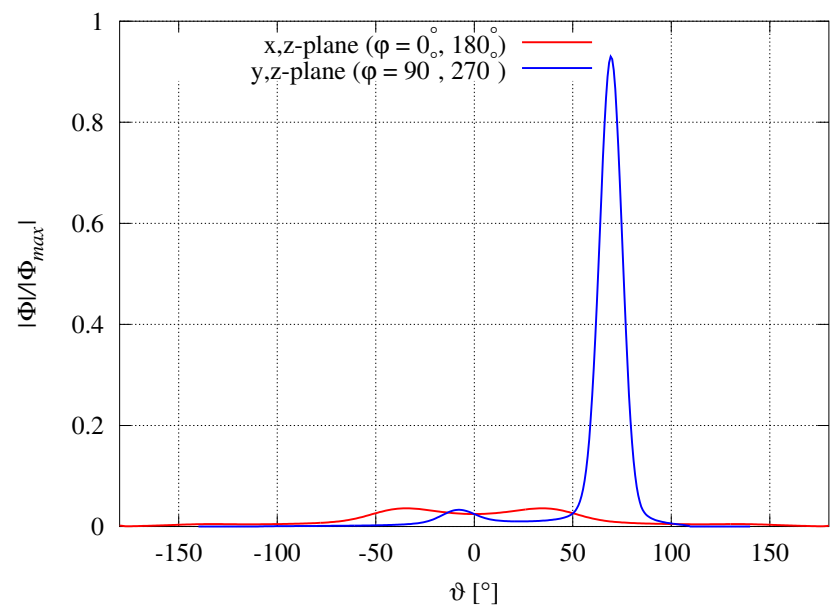

Figure 11. Radiation pattern of the scattered far field for an acoustically soft sector in the $x z$ and $y z$ plane. The source has the complex location $r_{c}=(0.001+10 j) \Lambda, \vartheta_{c}=90^{\circ}, \varphi_{c}=90^{\circ}$, that is $\boldsymbol{r}_{c}=(0.001+10 j) \Lambda \hat{x}$ in Cartesian coordinates.

propagates towards the corner in a plane of incidence parallel to the sector. For the acoustically soft sector (left-hand side) the beam is partly is reflected in $z$ direction. However, for the acoustically hard sector (right-hand side) the beam passes the sector completely unaffected.

\subsection{Scattered far fields}

The results for the scattered far fields (radiation patterns) are obtained by means of the large-argument approximation of the spherical Hankel functions of the second kind and by subtracting the incident field from the total field, see Eq. (27). Figures 6-8 show the radiation patterns of an acoustically soft sector for different angles of incidence $\varphi_{c}$.

For $\varphi_{c}=0^{\circ}$ (Fig. 6) maxima of the scattered field can be observed in the direction of propagation as well as in the opposite direction which is due to reflection. The pattern is nearly symmetric to the $y z$ as well as to the $x z$ plane.

For $\varphi_{c}=45^{\circ}$ (Fig. 7) we also see these two maxima mentioned before with the reflected part being slightly larger.

For $\varphi_{c}=90^{\circ}$ (Fig. 8) there appears a broad maximum in $y$ direction.

Figure 9 shows a pattern for an acoustically hard sector for a point source located at $\varphi_{c}=0^{\circ}$. In contrast to the result obtained for the acoustically soft sector (Fig. 6) the pattern is not symmetric to the $y z$ plane.

Finally, Figs. 10 and 11 represent radiation patterns of the scattered far fields of an acoustically soft sector in the $x z$ and $y z$ plane for different locations of the incident CSB waist.

\section{Conclusions}

It has been shown that a combined complex-source beam and spherical-multipole expansion allows to investigate the 
scattering and diffraction of a localized plane wave by the corner of an acoustically soft or hard plane angular sector by means of a convergent and numerically stable analysis.

Future work will include solutions for the corresponding electromagnetic problems, the investigation of the CSB scattering and diffraction by more general elliptic cones, and the investigation and development of techniques to integrate the findings into asymptotic methods.

Acknowledgements. This work was supported by the Deutsche Forschungsgemeinschaft (KL815/10-1\&2).

Edited by: U. van Rienen

Reviewed by: two anonymous referees

\section{References}

Abramowitz, M., and Stegun, I. A. (Eds.): Handbook of Mathematical Functions with Formulas, Graphs, and Mathematical Tables, New York, Dover Publications, p. 365, 1972.

Blume, S., Klinkenbusch, L., and Uschkerat, U.: The radar cross section of the semi-infinite elliptic cone, Wave Motion, 17, 365389, 1993.

Blume, S., and Klinkenbusch, L.: Spherical-multipole analysis in electromagnetics, in: Frontiers in Electromagnetics, edited by: Werner, D. H. and Mittra, R., New York, IEEE Press and Wiley, 553-608, 1999.

Bowman, J. J., Senior, T. B. A., and Uslenghi, P. L. E. (Eds.): Electromagnetic and acoustic scattering by simple shapes (revised printing), New York, Hemisphere Pub. Corp., 129-179, 1987.

Brüns, H. and Klinkenbusch, L.: Electromagnetic diffraction and scattering of a complex-source beam by a semi-infinite circular cone, Adv. Radio Sci., 11, 31-36, doi:10.5194/ars-11-31-2013, 2013.

Eisenhart, L. P.: Separable systems of Stäckel, Ann. Math., 35, 284305, 1934.

Eisenhart, L.P.: Stäckel systems in conformal euclidean space, Ann. Math., 36, 57-70, 1935.
Hansen, T. B.: Corner diffraction coefficients for the quarter plane, IEEE T. Antenn. Propag., 39, 976-984, 1991.

Hill, C. H.: A UTD solution to the EM scattering by the vertex of a perfectly conducting plane angular sector, $\mathrm{Ph} . \mathrm{D}$. dissertation, The Ohio State University, Columbus (OH), 1990.

Jansen, J. K. M.: Simple-periodic and non-periodic Lamé functions and their application in the theory of conical waveguides, Ph.D. dissertation, Eindhoven University of Technology, Eindhoven, The Netherlands, 1976.

Katsav, M., Heyman, E., and Klinkenbusch, L.: Complex-source beam diffraction by an acoustically soft or hard circular cone, Proc. International Conference on Electromagnetics in Advanced Applications (ICEAA 2012), Cape Town, South Africa, 2-7 September, 135, 2012.

Keller, J. B.: The Geometrical Theory of Diffraction, Proc. of the Symp. on Microwave Optics, Eaton Electronics Laboratory, McGill University, Montreal, Canada, June 1953, Vol. 1; Reprinted as "The Geometric Optics Theory of Diffraction", The McGill Symp. on Microwave Optics, edited by: Karasik, B. S. and Zucker, F. J., AFCRC, Bedford, MA, 2, 207-210, 1959.

Keller, J. B.: Geometrical theory of diffraction, J. Opt. Soc. Am., 52, 116-130, 1962.

Klinkenbusch, L.: Electromagnetic scattering by semi-infinite circular and elliptic cones, Radio Sci., 42, RS6S10, 2007.

Kouyoumjian, R. G. and Pathak, P. H.: A uniform geometrical theory of diffrcation for an edge in a perfectly conducting surface, Proc. IEEE, 62, 1448-1461, 1974.

Kraus, L.: Diffraction by a plane angular sector, Ph.D. dissertation, New York University, New York, 1955.

Maci, S., Tiberio, R., and Tocafondi, A.: Diffraction by a plane angular sector, J. Electromagnet. Wave, 8, 1247-1276, 1994.

Maci, S., Albani, M., and Capolino, F.: ITD formulation for the currents on a plane angular sector, IEEE T. Antenn. Propag., 46, 1318-1327, 1998.

Moon, P. and Spencer, D. E.: Field theory handbook (2nd ed.), Berlin, Heidelberg, New York, Springer Verlag, 136-143, 1971.

Radlow, J.: Diffraction by a quarter plane, Arch. Ration. Mech. An., 8, 139-158, 1961.

Satterwhite, R. S.: Diffraction by a plane angular sector, Ph.D. dissertation, The Ohio State University, Columbus (OH), 1969. 